\title{
Blind and Sparsity Level Adaptive Reconstruction of Wideband Time-frequency-varying Signals with Sub-Nyquist Sampling
}

\author{
Weichao Sun ${ }^{1, a}$, Zhichao Sha ${ }^{1, b}$, Xiang Wang $^{1, \mathrm{c}}$, Fenghua Wang $^{1}$, Zhitao \\ Huang $^{1}$ \\ ${ }^{1}$ College of Electronic Science and Engineering, National University of Defense Technology, \\ Changsha, Hunan, 410073, P.R.China \\ asweichao1266@163.com, bshazhichao_163@163.com, chhristopherwx@163.com
}

Keywords: Compressed sensing, sub-Nyquist sampling, sparsity level adaptive, modulated wideband converter, iterative adaptive approach.

\begin{abstract}
This paper addresses the problem of wideband time-frequency-varying signal sub-Nyquist sampling and reconstruction based on compressed sensing (CS) framework. We propose a system of blind and sparsity level adaptive signal reconstruction for wideband signals with sub-Nyquist sampling. We utilize modulated wideband converter (MWC) that deals well with multi-band signals to acquire sub-Nyquist samples, change the signal sensing and reconstruction model to parameters estimation model in the array signal processing, and apply iterative adaptive approach (IAA) to recover spectral support and reconstruct signals simultaneously without any prior knowledge. Simulation results show that the proposed method outperforms the continue to finite (CTF) following MWC in low signal-to-noise ratio (SNR).
\end{abstract}

\section{Introduction}

A major challenge in wideband spectrum sampling is the requirement of a high sampling rate which may exceed today's best analog-to-digital converters (ADCs) front-end bandwidths. Moreover, such high sampling rate will generate a large number of samples to process, affecting speed and power consumption [1].

To overcome the rate bottleneck, a number of compressed sensing (CS) [2,3] structures proposed for sub-Nyquist sampling enable sampling below Nyquist rate, without (or with little) sacrificing reconstruction quality [4]. The modulated wideband converter (MWC) [5,6] is one of the most successful structures. It can not only treat multiband signals, but be realized by off-the-shelf commercial components.

As the sparsity level of time frequency varying signal is often unknown, it will be a challenge of using CS in practical sensing hardware. Following MWC, a block entitled continue to finite (CTF) [5] is proposed to recover the spectral support and reconstruct signals from MWC compressive samples and it works well with continue time signals. Unfortunately, it must need a band number (sparsity level) priori or set a maximum band number (superfluous signals will be ignored). Moreover, the method that uses Moore-Penrose pseudoinverse to reconstruct signal is sensitive to noise in dealing time-frequency-varying signal conditions.Recently, an alternative data-dependent approach [7] has been proposed for signals exhibiting a sparse representation in array signal processing. In [8], the so-called iterative adaptive approach (IAA), which is nonparametric, hyperparameter, free-weighted, and least squares-based (LS), was proposed for passive sensing, range-doppler imaging, and channel estimation applications in array processing.

In this paper, we propose a blind and sparsity level adaptive reconstruction of wideband time-frequency-varying signals method that would also bring substantial saving in terms of the sampling rate. In the proposed method, we use MWC to acquire sub-Nyquist samples, and change the signal sensing and reconstruction model to parameters estimation model in array signal processing. Then, we apply IAA to recover spectral support and reconstruct signals simultaneously without any 
prior knowledge. Simulation results demonstrate that the method outperforms the CTF framework and has a perfect effect in low signal-to-noise (SNR).

\section{Modulated Wideband Converter}

As shown in Fig. 1, the modulated wideband converter (MWC) [6] consists of an analog front-end with $m$ channels. In the ith channel, the input signal $x(t)$ is multiplied by a periodic waveform $p_{i}(t)$, lowpass filtered, and then sampled at rate $f_{s}=1 / \mathrm{T}$.

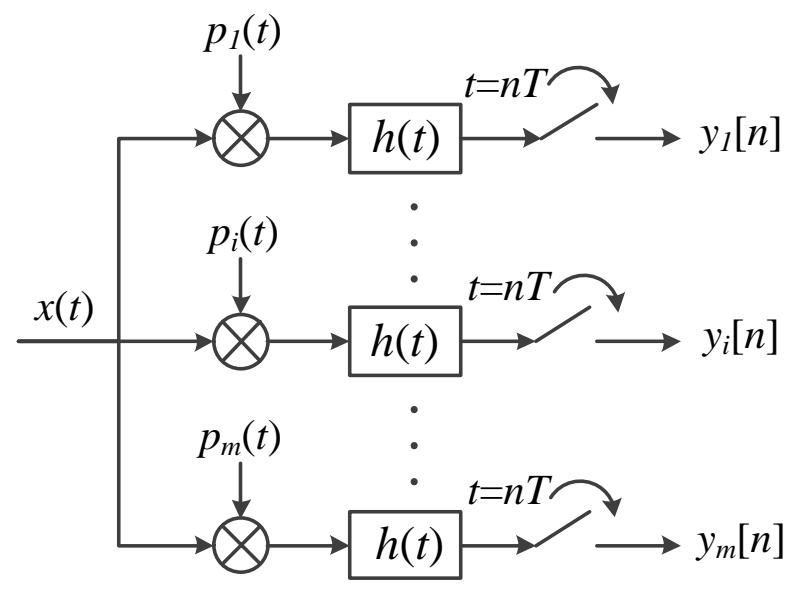

Fig.1 The modulated wideband converter structure

Consider the ith channel. Since pi(t) is Tp-periodic ( $T p=1 / f p)$, it has a Fourier expansion

$$
p_{i}(t)=\sum_{l=-\infty}^{\infty} c_{i l} e^{j 2 \pi l f_{p} t},
$$

where the coefficient $c_{i l}=\frac{1}{T_{p}} \int_{0}^{T_{p}} p_{i}(t) e^{-j 2 \pi l f_{p} t} d t$.

Then, the discrete-time Fourier transform (DTFT) of the ith sequence yi[n] is

$$
Y_{i}\left(e^{j 2 \pi f T}\right)=\sum_{l=-L_{0}}^{L_{0}} c_{i l} X\left(f-l f_{p}\right), \quad f \in[-1 / 2 T, 1 / 2 T]
$$

where $L_{0}$ is the smallest integer satisfying $L=2 L_{0}+1 \geq f_{\text {nyq }} / f_{p}, f_{\text {nyq }}$ is the Nyquist rate of $x(t)$. Therefore, $Y_{i}\left(e^{j 2 \pi f T}\right)$ is a linear combination of $f_{p}$-shifted copies of $X(f)$.

It is convenient to rewrite Eq. (2) in matrix form

$$
\mathbf{Y}(f)=\mathbf{A Z}(f), \quad f \in[-1 / 2 T, 1 / 2 T] .
$$

For each $f \in[-1 / 2 T, 1 / 2 T], \mathbf{Y}(f)$ is a vector of length $m$ with the ith element $Y_{i}(f)=Y_{i}\left(\mathrm{e}^{j 2 \pi f T}\right)$ which is discrete time Fourier transform (DTFT) of $y_{i}[n]$. The unknown vector $\mathbf{Z}(f)$ is of length $L$ with the lth element given by $\mathrm{Z}_{l}(f)=X\left(f+l f_{p}\right)$, where $X(f)$ is the Fourier transform of $x(t),-L_{0} \leq l$ $\leq L_{0}$. The $m \times L$ sensing matrix A consists of the coefficients $A_{i l}=c_{i,-l}=c_{i l}^{*}, 1 \leq i \leq m$.

\section{WMC with Iterative Adaptive Approach}

For the sake of simplicity, we choose $f_{s}=f_{p}$ in MWC parameters. We use inverse-DTFT for both side of Eq. 3, and then we get measurement function in time domain

$$
\mathbf{y}[k]=\mathbf{A z}[k], \quad k=1,2, \ldots, K,
$$


where $\mathbf{y}[k]=\left[y_{1}^{T}[k], y_{2}^{T}[k], \ldots, y_{m}^{T}[k]\right]^{T}, y_{i}[k]$ is the ith channel MWC compressive sample sequences, and $\mathbf{z}[k]=\left[z_{1}^{T}[k], z_{2}^{T}[k], \ldots, z_{L}^{T}[k]\right]^{T}, z_{i}[k]$ is the inverse-DTFT of $Z_{i}(f), K$ is the snapshot number. So, the sequences $z_{i}[k]$ are generated at the input rate $f_{s}$.

In order to use iterative adaptive approach (IAA) to recover spectral support and reconstruct original signals adaptively, we relate the MWC sampling model to parameters estimation model in array signal processing. In the MWC sampling model, we can treat each frequency band $z_{l}[k]$ as independent signals with the same received frequency. The sensing matrix $\mathbf{A}=\left[\mathbf{a}_{1}, \ldots, \mathbf{a}_{L}\right]$ which is column conjugate symmetric is then changed to be the steer matrix in array model, where $\mathbf{a}_{l}$ is the lth column of A.

IAA solves Eq. 4 by minimizing the following LS cost function

$$
\sum_{k=1}^{K}\left\|\mathbf{y}[k]-\mathbf{a}_{l} z_{l}[k]\right\|_{Q_{1}^{-1}}^{2} l=1,2, \ldots, L,
$$

where $\|\mathbf{x}\|_{Q_{l}^{-1}}^{2} \square \mathbf{x}^{H} Q_{l}^{-1} \mathbf{x}$ and

$$
Q_{l}=\mathbf{R}-P_{l} \mathbf{a}_{l} \mathbf{a}_{l}^{H}
$$

is the IAA interference and noise covariance matrix, where $P_{l}$ denotes the signal power at grid point $l$

$$
P_{l}=\frac{1}{K} \sum_{k=1}^{K}\left|z_{l}[k]\right|^{2} \quad l=1,2, \ldots, L .
$$

The IAA covariance matrix $\mathbf{R}$ has the expression

$$
\mathbf{R}=\mathbf{A P A}^{H}
$$

where $\mathbf{P}$ is a diagonal matrix with diagonal entries from the vector $\left[P_{1}, \ldots, P_{L}\right]^{T}$.

Using Eq. 6 and the matrix inversion lemma, minimizing Eq. 5 with respect to $z_{l}[k], k=1,2, \ldots, K$, yields the estimation of $z_{l}$

$$
\tilde{z}_{l}[k]=\frac{\mathbf{a}_{l}^{H} \mathbf{R}^{-1} \mathbf{y}[k]}{\mathbf{a}_{l}^{H} \mathbf{R}^{-1} \mathbf{a}_{l}}, \quad l=1,2, \ldots, L .
$$

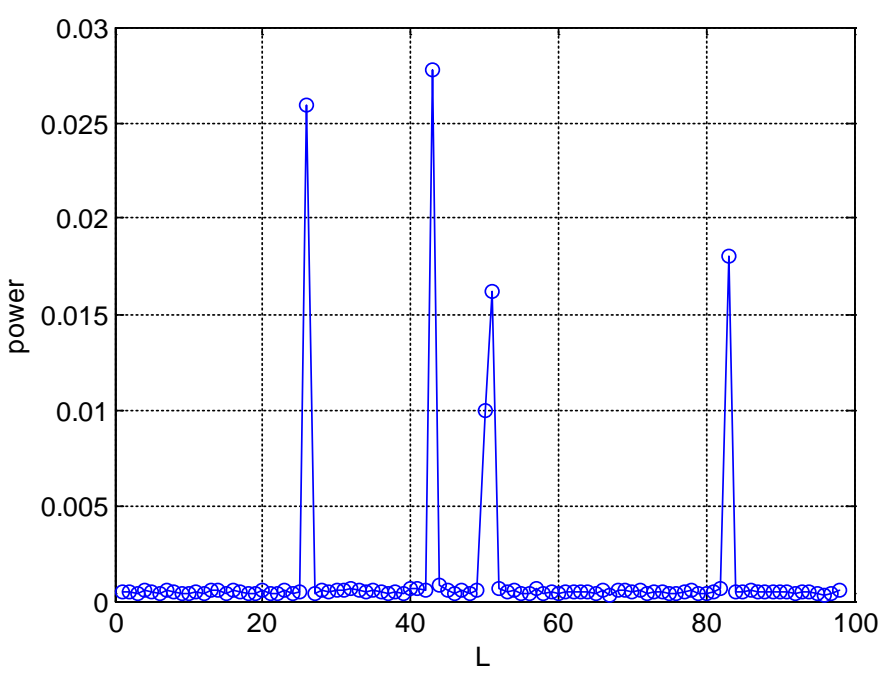

Fig.2 The power spectrum distribution estimated by MWC-IAA

Fig. 2 plots the signal power $P_{l}$ at different grid point. From Fig. 2, we can easily find the signal spectral support $S$ (the five indexes that have maximum values). Our empirical experience is that 
MWC-IAA does not provide significant improvements in performance after about 6 iterations. Once the support is found, the signal sampled at based band can be acquired

$$
\begin{array}{ll}
z_{l}[k]=\tilde{z}_{l}[k], & l \in S \\
z_{l}[k]=0, & l \notin S
\end{array}
$$

In order to get the original signal, we only need to interpolated $z_{l}[k]$ to the Nyquist rate and modulate it the original band indexed by the support $S$.

\section{Numerical Results}

Computer simulation is presented in this section to verify the performance of the proposed method compared with CTF framework [9].

In the following experiment, the number of potentially active transmissions $N_{\text {sig }}$ is set to be six. Each transmission is PSK modulation with two side bandwidth not than $50 \mathrm{MHz}$, random transmitting time and duration. Their carrier frequencies are drawn uniformly at random between $[0$, 5] $\mathrm{GHz}$, namely, the Nyquist sampling rate $f_{\text {nyq }}$ is at least $10 \mathrm{GHz}$. We choose channel number $m=50$, mixing function period $f_{p}=51.28 \mathrm{MHz}, L=195$ (calculated by $f_{\text {nyq }} / f_{p}$ ), each channel sampling rate $f_{s}=f_{p}$ and samples number is $K=90$. The received signal is polluted by Gaussian noise which is added and scaled so that the test signal has the desired signal-to-noise ratio (SNR). In the experiment, we assume that the MWC-CTF has a band number priori, but the proposed MWC-IAA still has no priori. Each experiment is repeated over 500 realizations.

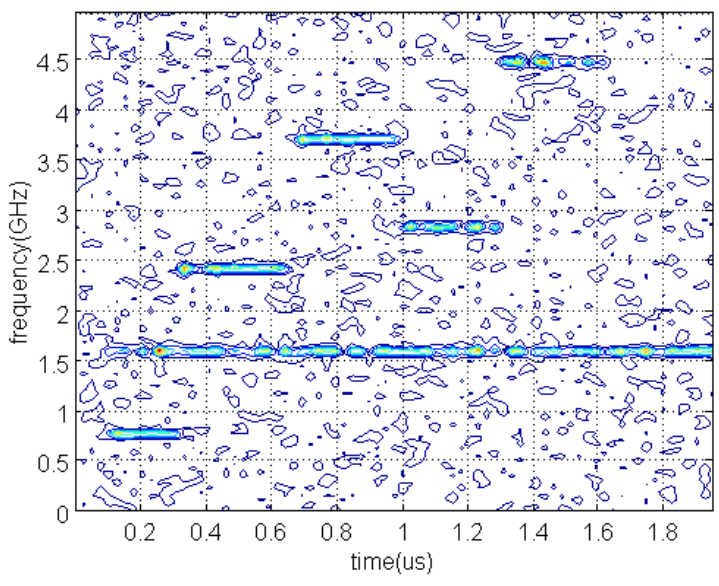

(a) The STFT of original signal

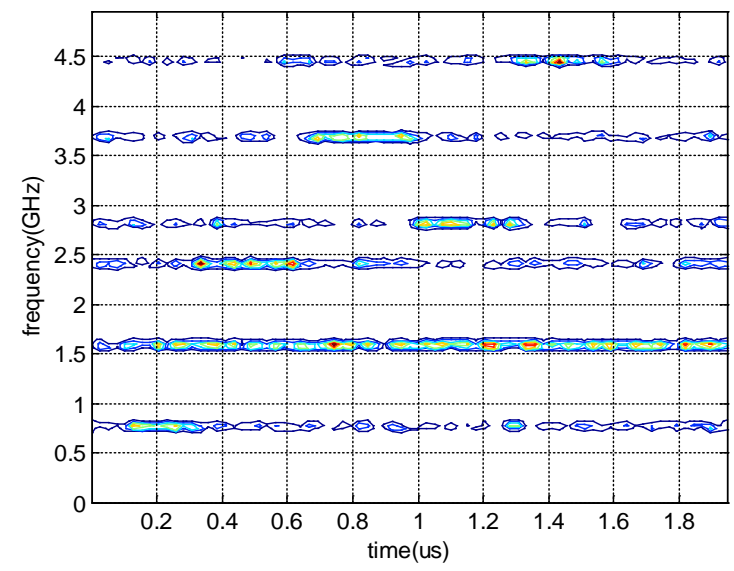

(b) The STFT of signal reconstructed by MWC-CTF 


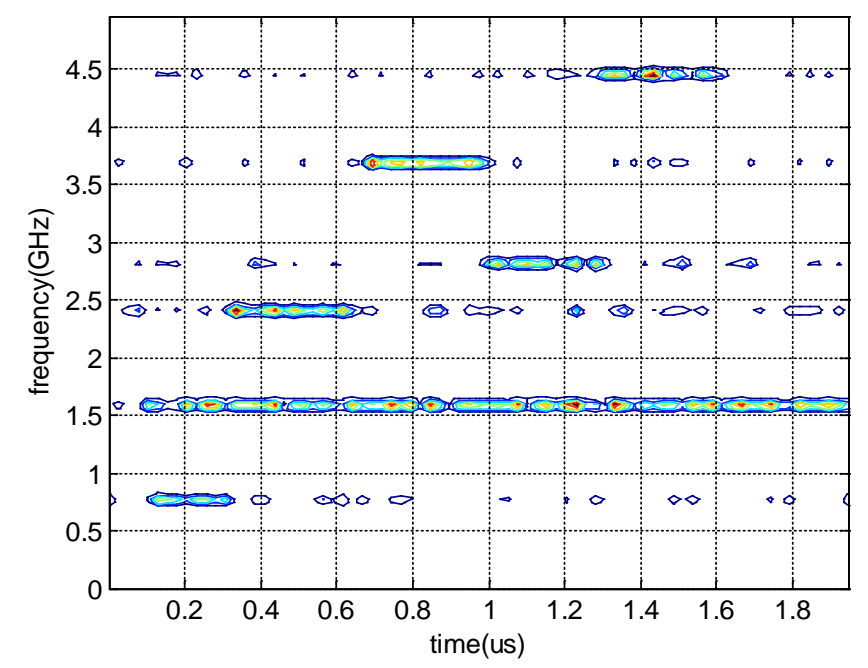

(c) The STFT of signal reconstructed by MWC-IAA

Fig.3 The STFT of (a) original signal (b) signal reconstructed by MWC-CTF (c) signal reconstructed by MWC-IAA with SNR=-5dB

In Fig. 3, there are six time-frequency-varying signals with different starting time, time duration and frequencies in the SNR $=-5 \mathrm{~dB}$ condition. Fig. 3 plots the STFT of original signal, reconstructed signal by MWC-CTF and MWC-IAA respectively. Clearly, in low SNR conditions, the MWC-CTF has reconstructed false frequencies at the free time of the signal transmission or brought in more noise. If we have no band number priori, the result will be much worse. Comparatively, the proposed MWC-IAA has almost perfectly reconstructed the signal at the right time and frequencies with less noise.

In order to evaluate the quality of reconstruction, we apply a criterion named similarity degree [10]

$$
\mathrm{R}(\tilde{u}, u)=\frac{\tilde{u}^{H} u}{\|\tilde{u}\|\|u\|},
$$

where $\tilde{u}$ is the estimation of $u$. If $\tilde{u}$ approaches to $u$, the similarity degree comes near to 1 . On the contrary, the degree approaches to 0.

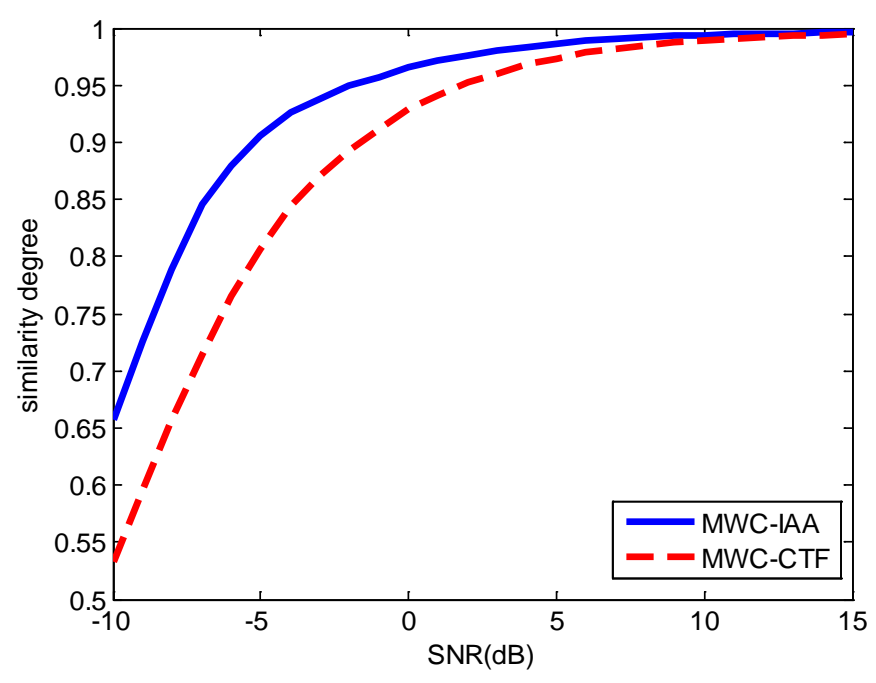

Fig.4 The similarity degree comparison 
Fig. 4 plots the similarity degree between reconstructed signal (by proposed MWC-IAA and MWC-CTF respectively) and original signal (without noise) with different SNR. As can be seen from this figure, both of the waveform similarity degrees increase with the SNR. It is clear that the reconstructed performance can be improved better under better SNR conditions. When the SNR is above $10 \mathrm{~dB}$, both of the two methods have a perfect performance. However, with the SNR decreasing, the proposed MWC-IAA method has a much better performance than MWC-CTF.

\section{Conclusion}

A system of blind and sparsity level adaptive signal sub-Nyquist sampling and reconstruction for wideband time-varying signals is proposed to mitigate the limitations of high sampling rate, prior knowledge and low SNR. The proposed technique utilizes MWC sampling scheme that can sampling sparse multi-band signal at low rate, turns the problems of spectrum sensing and reconstruction into parameter estimation in array signal processing and solves them by iterative adaptive approach. The final simulation results indicate that the proposed method outperforms CTF framework in low SNR.

\section{References}

[1] D. Cohen, Y.C. Eldar: IEEE Trans. Signal Process. Vol. 62-15 (2014), p. 3897

[2] D.L. Donoho: IEEE Trans. Inf. Theory Vol. 52-4 (2006), p. 1289

[3] E.J. Candes, J. Romberg and T. Tao: IEEE Trans. Inf. Theory Vol. 52-4 (2006), p. 489

[4] Y. You, J. Jin, W. Duan and et al: IEICE Electron. Express Vol. 9-4 (2012), p. 314

[5] M. Mishali and Y.C. Eldar: IEEE J. Sel. Topics Signal Process. Vol. 4-2 (2010), p. 375

[6] M. Mishali, Y.C. Eldar, O. Dounaevsky and et al: IET Circuits Devices Syst. Vol. 5-1 (2011), p. 8

[7] T. Yardibi, J. Li and P. Stoica: Asilomar Conf Signals Syst and Computers (2008)

[8] T. Yardibi, J. Li, P. Stoica and et al: IEEE Trans. Aerosp. Electron. Syst. Vol. 46-1 (2010), p. 425

[9] Information on http://webee.technion.ac.il/people/YoniaEldar/

[10] C. Bouder, S. Azou and G. Burel: J. Franklin Ins. Vol. 341-7 (2004), p. 595 